\title{
Impact of competing crystallization processes on the
} structure of all-conjugated donor-acceptor block

\section{copolymers P3HT-b-PNDIT2 in highly oriented thin films}

Viktoriia Untilova ${ }^{1}$, Fritz Nübling ${ }^{2}$, Laure Biniek ${ }^{1}$, Michael Sommer ${ }^{2}$ and Martin Brinkmann $^{1 *}$

(1) Institut Charles Sadron, CNRS-Université de Strasbourg, 23 rue du Loess, Strasbourg 67034, France

(2) Institut für Chemie, Technische Universität Chemnitz, Straße der Nationen 62, 09111 Chemnitz, Germany

(3) Freiburger Materialforschungszentrum, Stefan-Maier-Straße 21, 79014 Freiburg, Germany. 


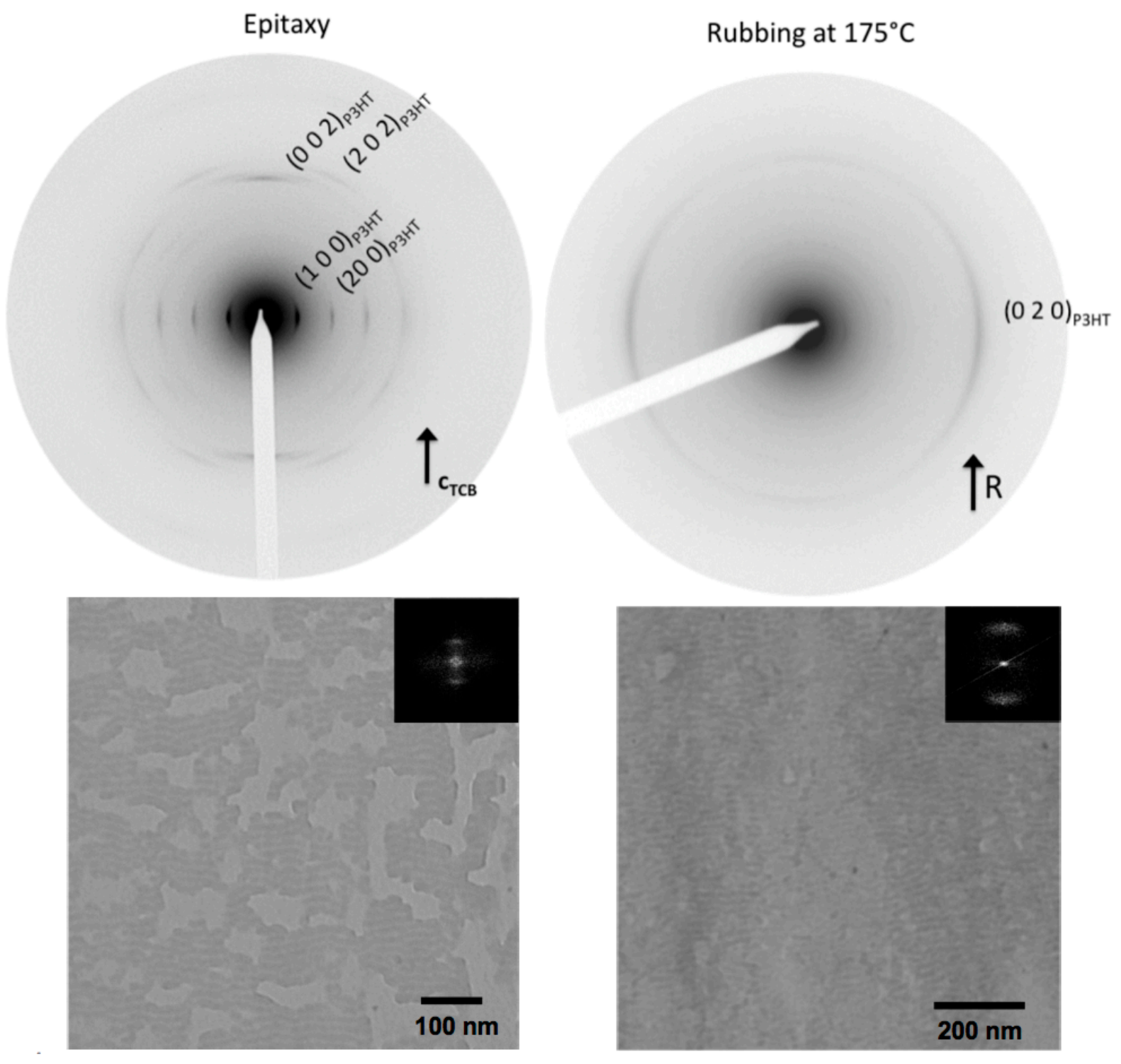

Figure S1. Comparison of the electron diffraction patterns and bright field of thin oriented films of the P3HT block $\left(\mathrm{M}_{\mathrm{n}}=7 \mathrm{kDa}\right)$ prepared by epitaxy and high temperature rubbing $\left(\mathrm{T}_{\mathrm{R}}=175^{\circ} \mathrm{C}\right)$. The arrows point at the resulting chain direction (parallel to $\mathrm{c}_{\mathrm{TCB}}$ for epitaxy on TCB or parallel to the rubbing direction $\mathrm{R})$. 

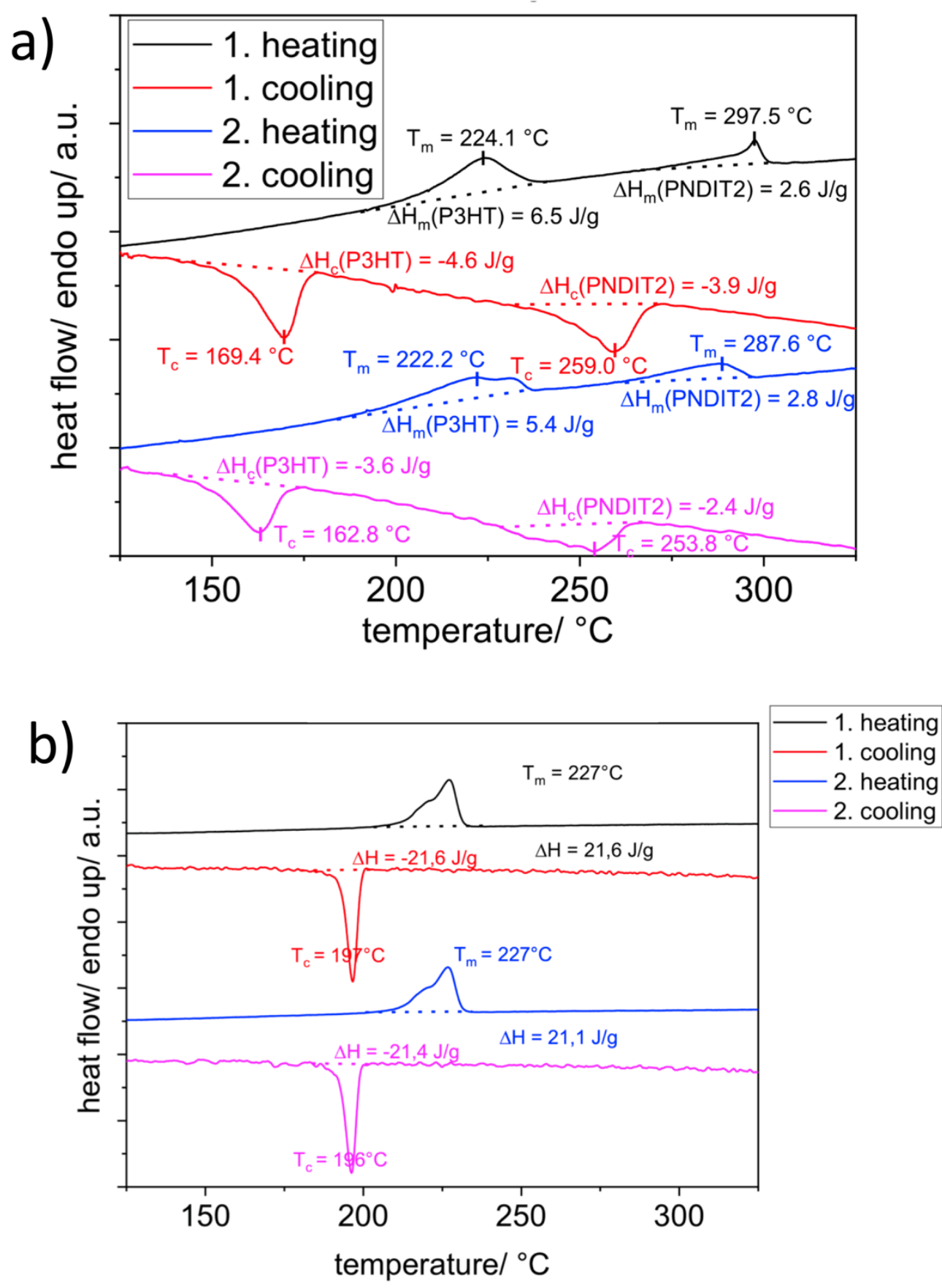

Figure S2. DSC traces of the P3HT- $b$-PNDIT2 copolymer (a) and of the P3HT block (b). 


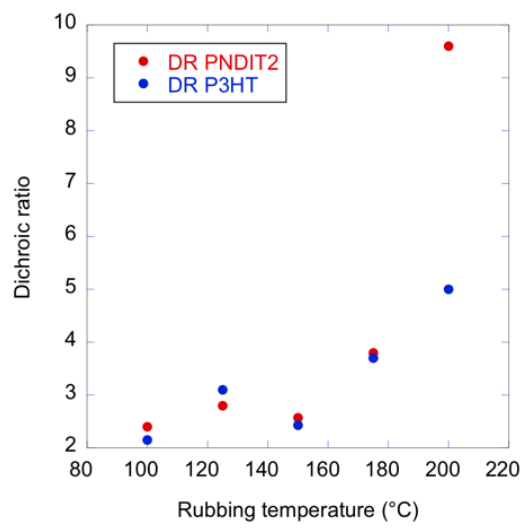

Figure S3. Variation of the dichroic ratio at two wavelengths $(695-701 \mathrm{~nm}$ for PNDIT2 and 553-558nm for P3HT) for P3HT- $b$-PNDIT2 copolymer films oriented by rubbing at different temperatures $\left(T_{R}\right)$.
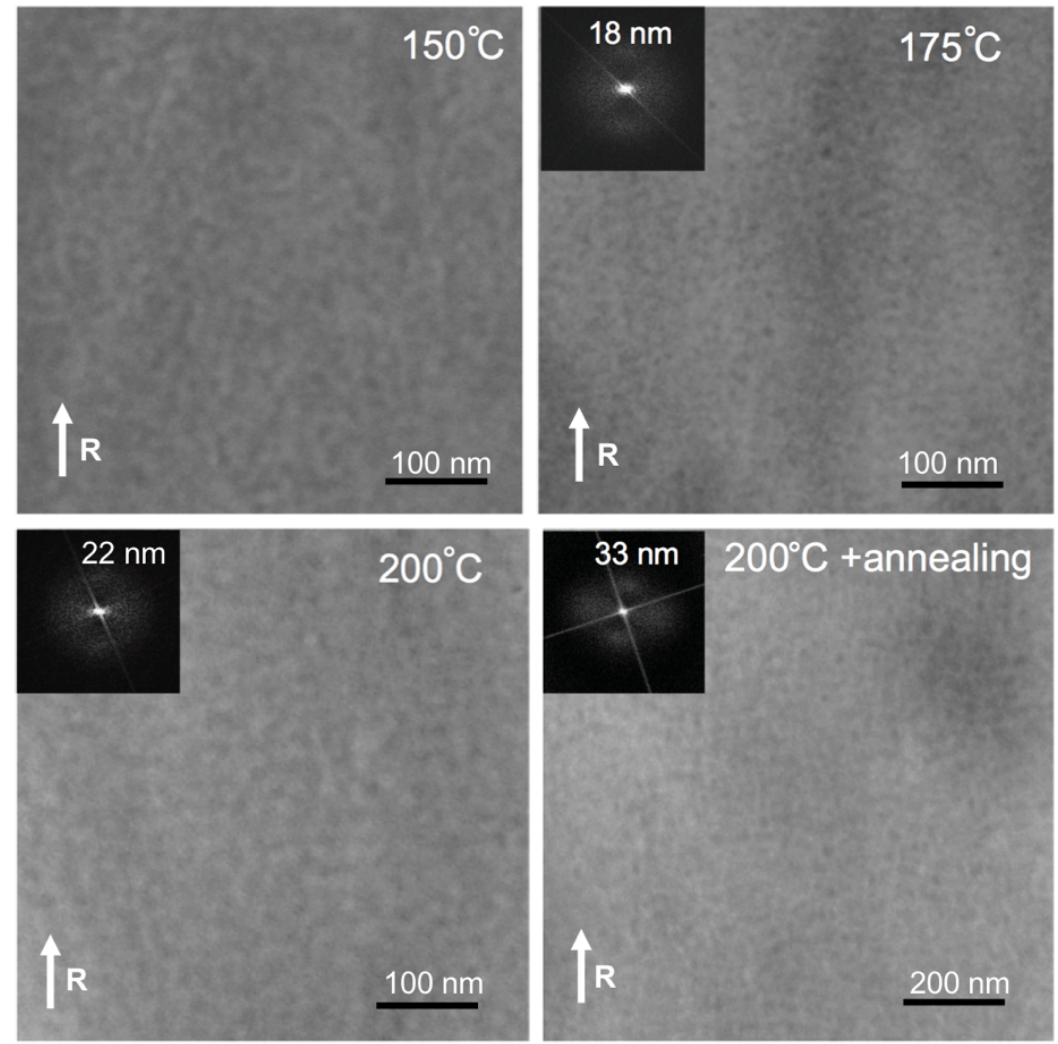

Figure S4. Bright Field TEM images of oriented P3HT- $b$-PNDIT2 thin films rubbed at different temperatures $\left(150^{\circ} \mathrm{C}, 175^{\circ} \mathrm{C}\right.$ and $\left.200^{\circ} \mathrm{C}\right)$ as well as for a film rubbed at $200^{\circ} \mathrm{C}$ and subsequently annealed at $250^{\circ} \mathrm{C}$. The insets correspond to the FFTs of the images with the measured total lamellar periodicity. 

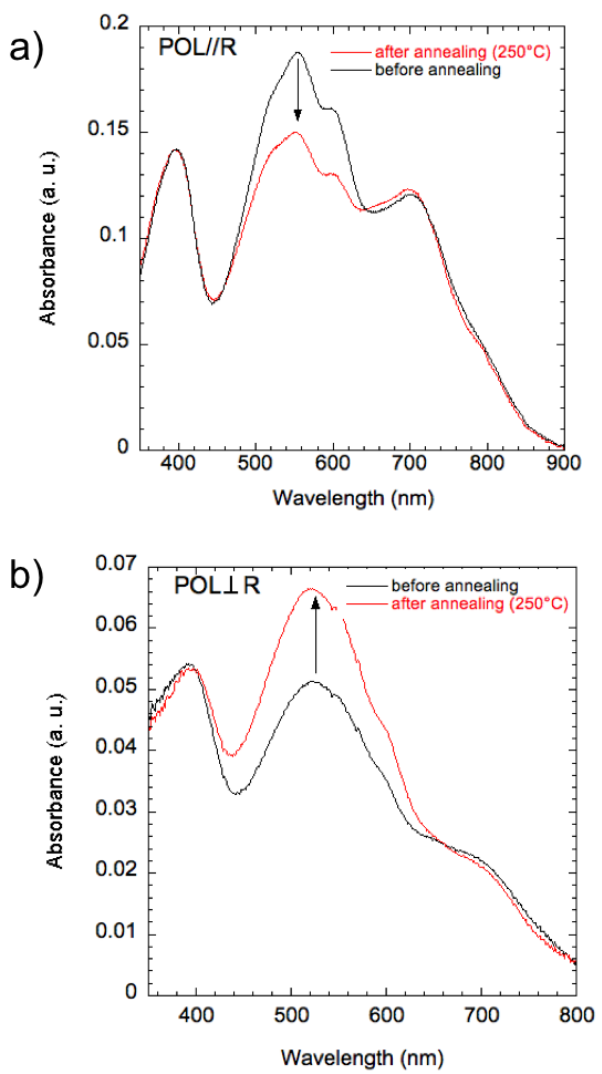

Figure S5. Evolution of the UV-vis spectra of oriented pNDIT2- $b$-P3HT copolymer rubbed at $200^{\circ} \mathrm{C}$ after thermal annealing at $\mathrm{T}_{\mathrm{A}}=250^{\circ} \mathrm{C}$. (a) Spectra for incident polarization POL parallel to the rubbing direction. (b) Spectra for incident polarization POL perpendicular to the rubbing direction. 